www.nature.com/pj

\title{
Nonlinear changes in pore size induced by temperature in the design of smart membranes
}

\author{
Rodolfo Fabian Estrada Guerrero ${ }^{1,2}$, Efrain Rubio Rosas ${ }^{2}$ and Ventura Rodriguez Lugo ${ }^{2}$
}

A new kind of synthetic membranes prepared by crosslinking of two different organic polymers was designed, the membranes has the ability to adjust the pore diameter in a wide range of sizes (from 3 to 100 microns) just by changing the time of thermal treatment in an aqueous media at constant temperature where they are immersed; in this way it is possible to control externally the pore size of the membrane. A nonlinear mathematical model, on the basis of the overlap of the electrical double layers of charged particles, was developed to explain the oscillatory behavior of the pore size when the time of thermal treatment was varied. The model deduced fits correctly to the experimental results and describes the oscillatory behavior of the pore size. Polymer Journal (2010) 42, 947-951; doi:10.1038/pj.2010.88; published online 13 October 2010

Keywords: nonlinear changes; pore size; smart membranes; thermal treatment

\section{INTRODUCTION}

The use of polymeric membranes for filtration and separation processes has a considerable number of applications as is in reverse osmosis, ${ }^{1-3}$ water purification, ${ }^{4}$ chemical and biological separations, ${ }^{5}$ gases separation, ${ }^{6,7}$ separation of heavy metals from a solution, ${ }^{8}$ templates for the growth of some biomaterials, ${ }^{9}$ among many others. Owing to these important applications, too much effort has been addressed to the design of new membranes with high selectivity and chemical resistance. Nowadays, it is possible to impose to the membranes an additional requirement related to the possibility to control their selectivity externally by changing some external parameters. $^{2}$

Cellulose acetate is one of the most commonly used material for membrane fabrication because it allows to produce membranes with adequate porosity and low binding. ${ }^{6}$ Nevertheless, very few efforts have been dedicated to produce membranes with porosity externally controlled. ${ }^{4}$

An important achievement is to have the possibility of controlling the pore size and its distribution. For this purpose, some specific compounds focused on the required specific pore size have been designed. Some of them are mixed with the polymer to form the membrane. Once the membrane has formed, it is subjected to a physical or chemical treatment in order to remove the compound used during the synthesis. This procedure allows to obtain membranes with a very specific pore size and the morphology is fixed.

In this work, the pore size of synthetic polymeric membranes made of cellulose acetate crosslinked with polyacrylic acid was determined after they were immersed in hot water keeping the temperature constant and varying the time of immersion. The results show that it is possible to control externally the membrane porosity just by varying the time of immersion in hot water.

The pore size shows a nonlinear oscillation as a function of the temperature. To explain this nonlinear behavior, a mathematical model based on the overlap of the electrical double layers of two charged particles that approach each other was used to fit the experimental data. For the deduction of the model three types of forces were included: (a) the repulsion force produced in the overlapping of the electrical double layers of charged polymer molecules, (b) the repulsion force due to the steric interaction produced by the overlapping of the polymer molecules caused by the swelling process and (c) the elastic force produce by changes in the conformational entropy of the molecule. With these terms it was possible to obtain an analytical expression for the dependence of the pore size with the time of immersion; using this expression, the experimental data were well fitted. Mathematical details are given in the Appendix.

\section{EXPERIMENTAL PROCEDURE}

In order to carry out this work, two lots of membranes named M38 and M39 were prepared as reported elsewhere. ${ }^{10}$ For the lot M38, a solution was prepared dissolving $8 \mathrm{~g}$ of cellulose acetate (Fluka Chem, Bruchs, Switzerland) in $100 \mathrm{ml}$ of glacial acetic acid (Sigma-Aldrich, St Louis, MO, USA) at room temperature; when the cellulose acetate has been completely dissolved, $10 \mathrm{ml}$ of polyacrylic acid with a molecular weight of $30000 \mathrm{~g} \mathrm{~mol}^{-1}$ is added slowly with constant medium agitation; this solution was heated at $60^{\circ} \mathrm{C}$ under agitation for $30 \mathrm{~min}$, allowing the crosslinking reaction between the cellulose acetate and the polyacrylic acid to take place. The solution was cooled down to room temperature and stored for 3 days at atmospheric pressure in a dark place to avoid the influence of sunlight on the solution. To obtain the membranes, 10 samples of $10 \mathrm{ml}$ each were poured into flat glass molds of $10 \mathrm{~cm}$ in diameter,

\footnotetext{
${ }^{1}$ Departamento de Física y Matemáticas, Universidad Iberoamericana, México, Alvaro Obregón, México and ${ }^{2}$ Centro Universitario de Vinculación, Benemérita Universidad Autónoma de Puebla, Ciudad Universitaria, Puebla, México

Correspondence: Dr RFE Guerrero, Department of Physics and mathematics, Universidad Iberoamericana, Prolongación Paseo de la Reforma 880, Lomas de Santa Fé, México D.F, Alvaro Obregón 01219, Mexico.

E-mail: rodolfo.estrada@uia.mx
}

Received 10 May 2010; accepted 12 August 2010; published online 13 October 2010 
leaving the molds with the solution flouting on iced water at $4{ }^{\circ} \mathrm{C}$ for $30 \mathrm{~s}$, allowing the solution to reach the same temperature of the iced water. Thereafter, the mold with the polymer solution was completely immersed carefully into the cold water until the membranes were formed and permitted to rest for $15 \mathrm{~min}$ to allow the solidification of the polymer solution. Once the membranes have been formed they were withdrawn from the iced water and immediately placed into a bath of hot water at $60^{\circ} \mathrm{C}$. The time of treatment into the hot water varied from 0.5 to $4.5 \mathrm{~min}$ at regular intervals of $0.5 \mathrm{~min}$. One of the membranes was used as reference and did not receive treatment in hot water.

The lot of membranes labeled M39 was prepared dissolving $6 \mathrm{~g}$ of cellulose acetate in $100 \mathrm{ml}$ of glacial acetic acid and following the same procedure as the lot M38.

The pore size was determined with a scanning electron microscope Jeol JSM6610LV (Jeol, Peabody, MA, USA) operated in the high vacuum mode at an acceleration voltage of $20 \mathrm{kV}$ and a pressure of $20 \mathrm{~Pa}$, the membranes were previously coated with gold. Both lots of membranes were characterized by infrared spectroscopy in a Bruker spectrometer (Bruker, Karlsruhe, Germany).
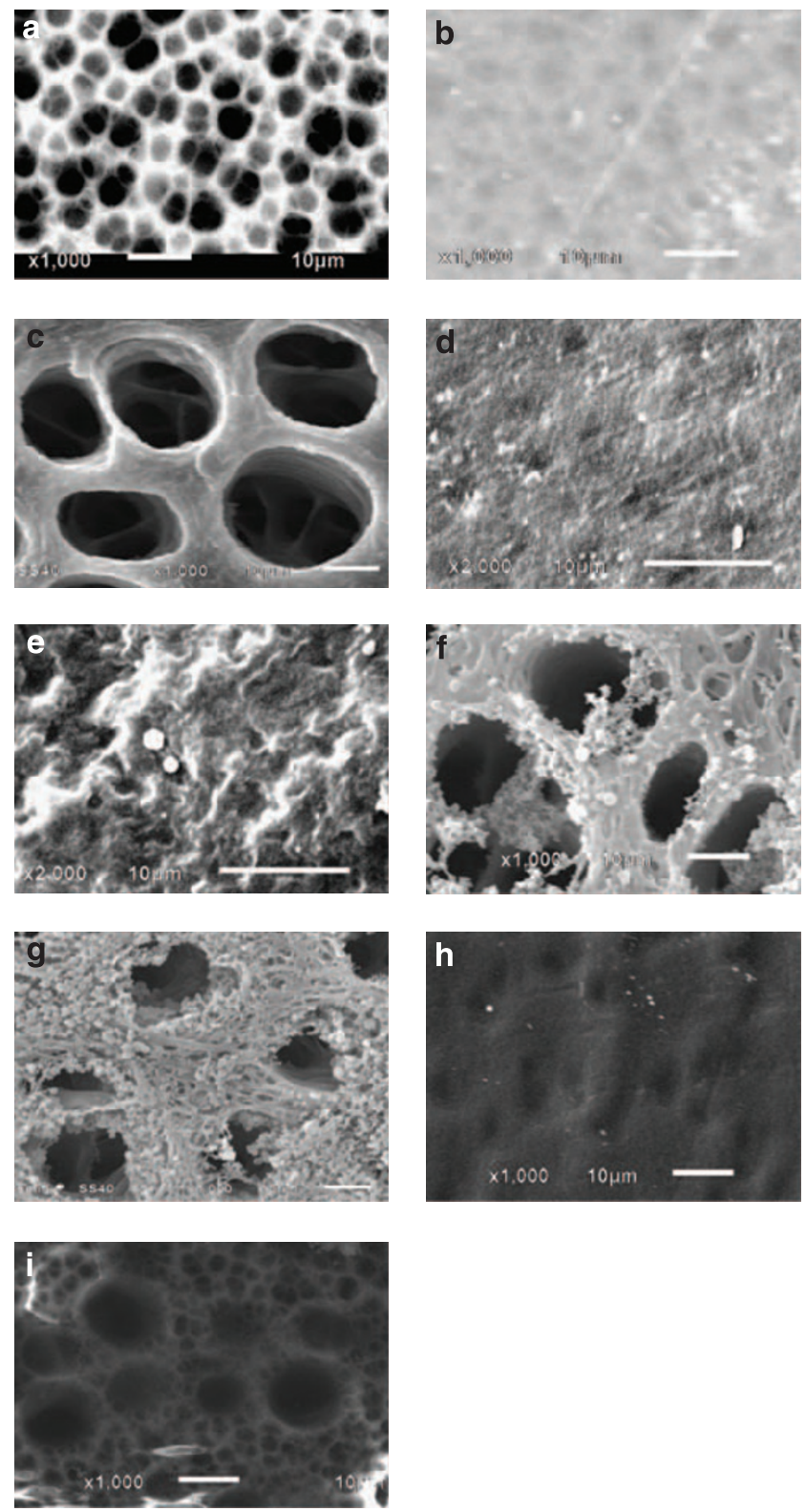

Figure 1 Sequence of scanning electron microscope images for the lot M38 showing the variation of the pore diameter. (a) Membrane without thermal treatment, (b-i) membranes with thermal treatment.

\section{RESULTS}

The images obtained from electron microscopy are shown in Figures 1 and 2. Figure 1a corresponds to the membrane of the lot M38 without thermal treatment, Figures $1 \mathrm{~b}-\mathrm{i}$ correspond to the lot M38 with thermal treatment at different times. Figure 2a is the image of the membrane without thermal treatment for the lot M39, Figures $2 \mathrm{~b}-\mathrm{i}$ correspond to the lot M39 with thermal treatment at different times.

In accordance with the results obtained from the microscopic characterization, it can be seen that the pore size is changing depending on the time of immersion in hot water. In both lots of membranes, the pore begins with a specific size. As the time of thermal treatment progresses, the pore size is reduces and increases, showing an oscillatory behavior. In some membranes, the pores are formed in layers, giving the effect of depth forming
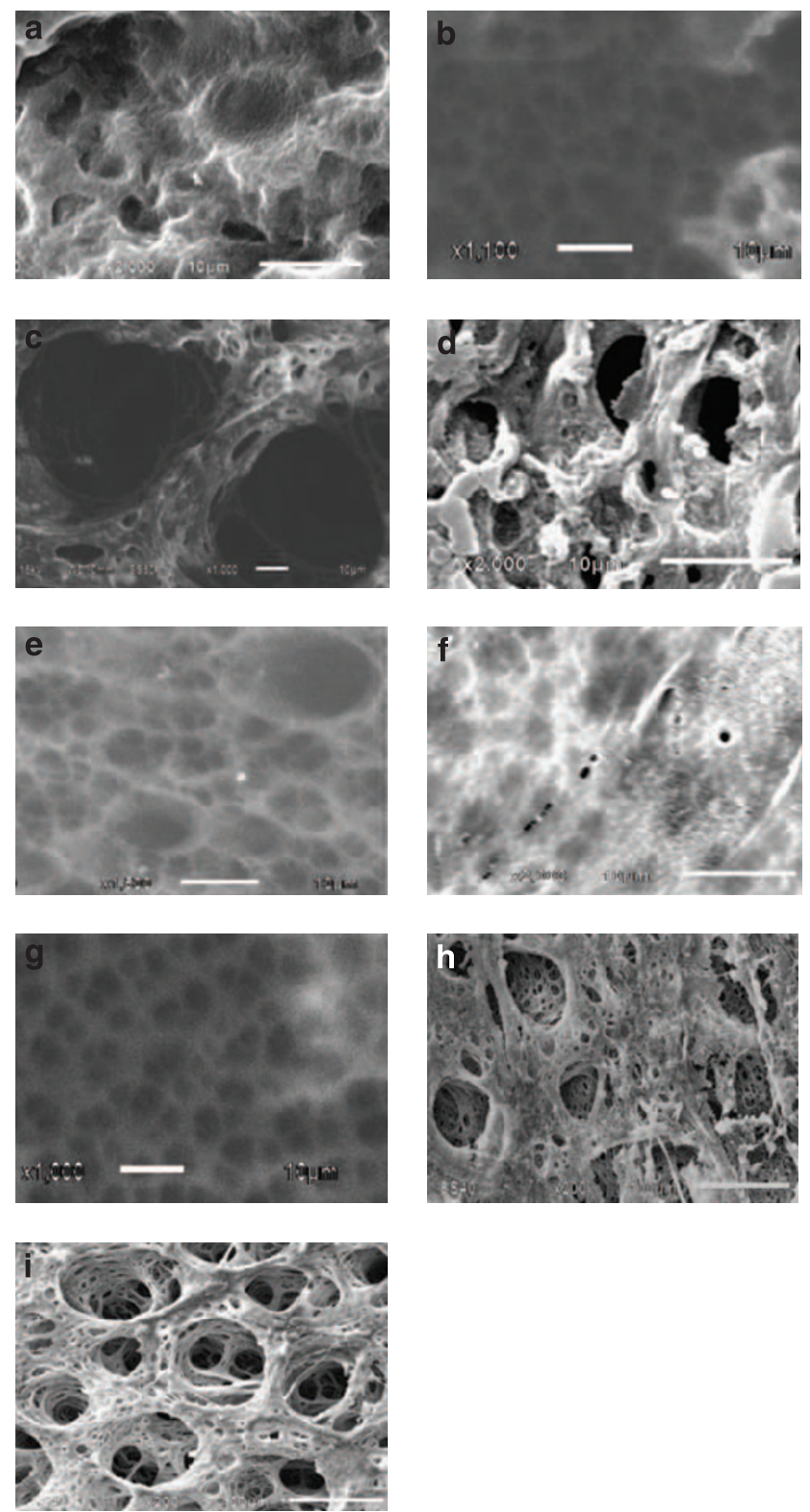

Figure 2 Sequence of scanning electron microscope images for the lot M39 showing the variation of the pore diameter. (a) Membrane without thermal treatment, (b-i) membranes with thermal treatment. 
a network; even the walls of the pores are porous as well. In Table 1, the results of this behavior are reported for both lots of membranes.

Table 1 Measurement of the variation in pore diameter for both lots of membranes

Lot M38 Lot M39

Immersion time ( $\mathrm{min}) \quad$ Pore diameter $(\mu \mathrm{m}) \quad$ Pore diameter $(\mu \mathrm{m})$ Thickness $(\mathrm{mm})$

\begin{tabular}{lrrl}
\hline 0 & 12.12 & 16.87 & 0.05 \\
0.5 & 3.40 & 6.00 & 0.05 \\
1.0 & 29.20 & 63.00 & 0.05 \\
1.5 & 2.90 & 12.30 & 0.05 \\
2.0 & 3.10 & 10.70 & 0.05 \\
2.5 & 30.20 & 12.30 & 0.05 \\
3.0 & 20.86 & 6.03 & 0.05 \\
3.5 & 3.50 & 47.31 & 0.05 \\
4.0 & 20.77 & 100.00 & 0.05 \\
\hline
\end{tabular}

a

Lot M38

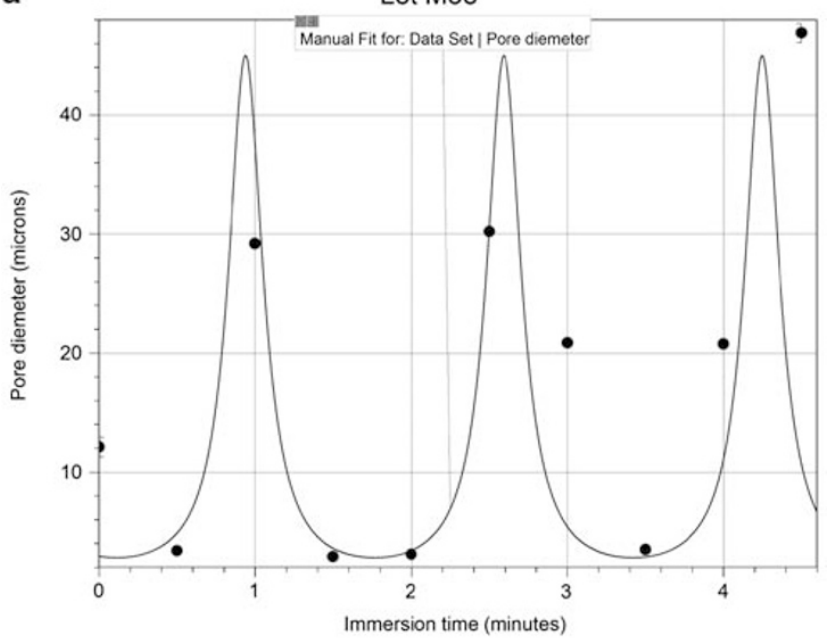

b

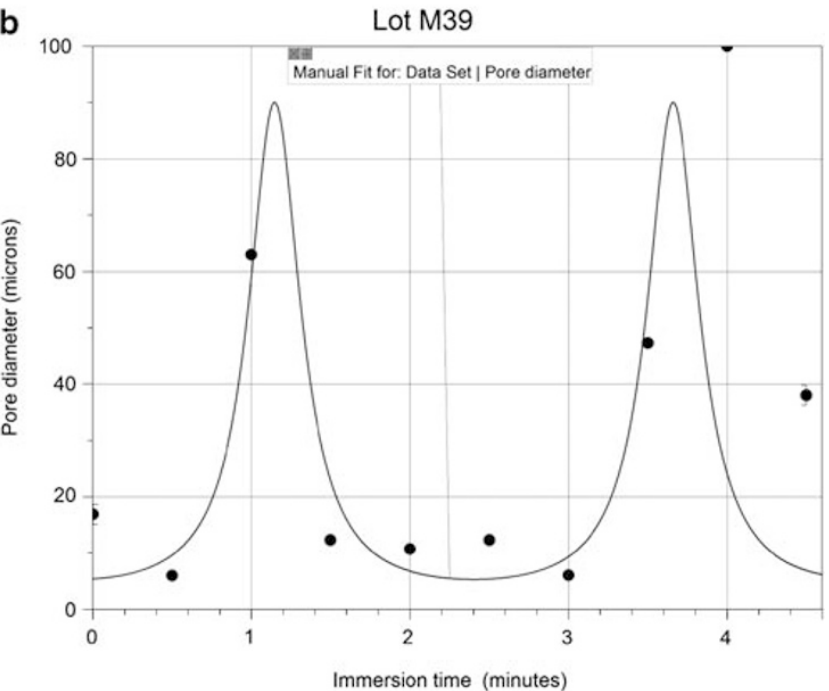

Figure 3 Plot of the experimental data and the fitting curve obtained applying the mathematical model deduced, (a) correspond to the lot M38, (b) correspond to the lot M39.
Figure 3 corresponds to the plot of the experimental data. Figure $3 \mathrm{a}$ is the plot of the data obtained for the lot of membranes M38, the black points are the experimental data, and the solid curve is obtained

a

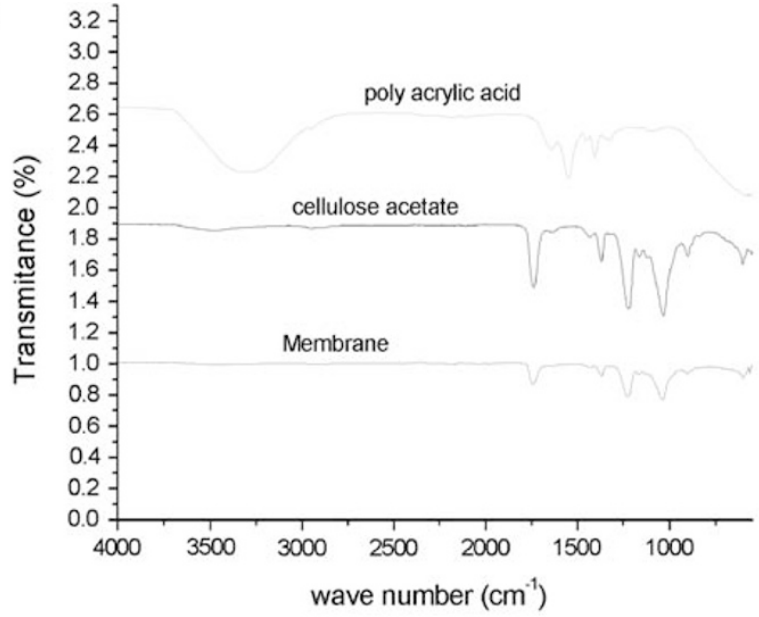

b

IR spectra Lot. M38
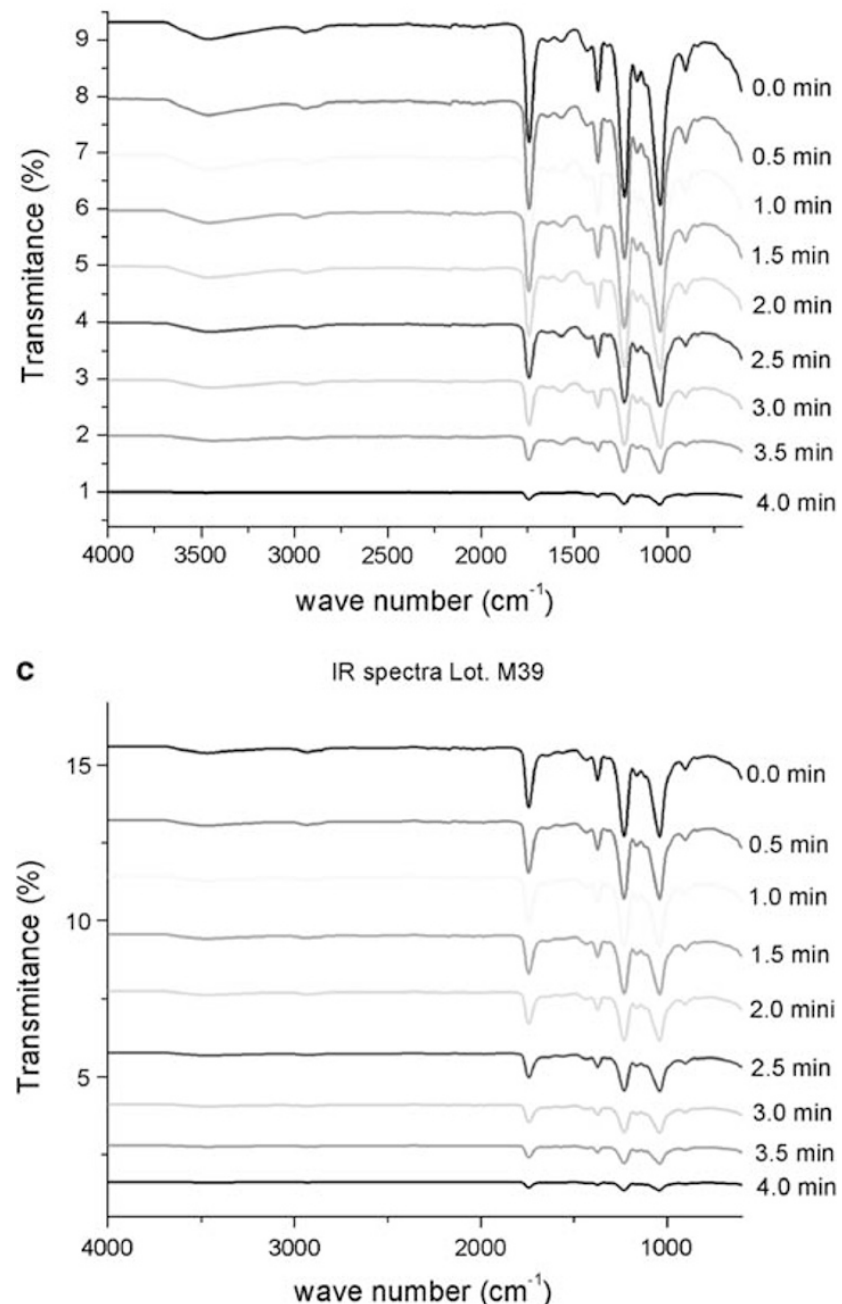

Figure 4 Infrared spectra. (a) Infrared (IR) of the raw materials, (b) sequence of IR spectra for the lot of membranes M38, (c) sequence of IR spectra for the lot of membranes M39. 
applying the mathematical model deduced. Figure $3 \mathrm{~b}$ is the plot of the experimental data (black points) for the lot of membranes M39, applying the mathematical model deduced to these points the fitting curve is obtained, as shown in the figure (solid curve). As can be noticed from both graphics, the pore size in both lots of membranes presents the same oscillatory behavior reducing and increase the diameter of the pores as the time of thermal treatment progresses. In both cases, one can appreciate that the mathematical model describes in a very good approximation the oscillatory behavior of the pore size as the time of thermal treatment progresses.

In Figure 4 the infrared spectra are presented. Figure $4 \mathrm{a}$ shows the infrared spectra of the raw materials used for the synthesis of the membranes. Figure $4 \mathrm{~b}$ displays the infrared spectra of the lot M38 and Figure $4 \mathrm{c}$ the infrared spectra of the lot M39. As can be noticed in both lots when the membrane has been formed the bands that corresponds to the carboxyl group in the polyacrylic acid and the bands corresponding to the acetate group in cellulose acetate disappears, this is due to the crosslink reaction between the polyacrylic acid and cellulose acetate that is carried out at this chemical groups.

\section{CONCLUSIONS}

The results obtained show that it is possible to design synthetic membranes whose pore size can be adjusted just by changing an external stimuli such as the time of thermal treatment while, keeping the external temperature constant. The variation in the pore size was in a wide range from 6 to $60 \mu$ when the time of thermal treatment was varied from 0.5 to $4.5 \mathrm{~min}$. The pore size depends in a nonlinear cyclic way with the time. The changes in pore size are due to electrostatic interactions and not by chemical reactions as is demonstrated by the Fourier transform infrared results. The mathematical model developed explains this nonlinear behavior and was based on three different mechanisms: (1) the forces produced by the overlapping of the electric double layers when two charged particles approach each other, (2) the forces produced by the overlapping of the polymer molecules when they are swelled by the medium applying the Flory-Krigbaum theory, (3) the elastic force due to conformational entropy changes. The experimental data were well fitted using this model. The design of these membranes open the possibility to use them in situations where the temperature is constant and the porosity needs to be opened and closed during the time in a particular application.

1 Osada, Y. \& Nakagawa, T. Membrane Science and Technology 239-358 (Marcel Deeker, USA, 1992).

2 Rodríguez, R. \& Castaño, V. M. Smart membranes: a physical model for a circadian behavior. Appl. Phys. Lett. 87, 1-3 (2005).

3 Ruiz-Treviño, F. A. \& Paul, D. R. A quantitative model for the specific volume of polymer diluents mixtures in the glassy state. J. Appl. Polym Sci. (Part B) 36, 1037-1050 (1998).

4 Merten, U. Desalination by Reverse Osmosis 55-89 (MIT, USA, 1966).

5 Bartsch, R. A. \& Way, J. D. Chemical Separation with Membranes 57-74 (American Chemical Society, ACS Symposium series, USA, 1996).

6 Park, S. \& Crank, J. Diffusion in Polymers 277-319 (Academic Press, NY, 1968).

7 Baker, R. W. Membrane Technology and Applications 2nd edn (J Wiley, NY, 2004).

8 Pinnau, I. Advanced Materials for Membrane Applications Vol. 1, 1-23, 106-128, and 269-280 (American Chemical Society, 2004).

9 Liuy, Y, Huang, S, Dan, X \& Zhou, K Growth of Hydroxyapatie crystals in presence of organic film. J. Mater. Sci. Technol. 20, 223-226 (2004).

10 Estrada, R. F., Rodriguez, R. \& Castaño, V. M. Smart polymeric membranes: self-control of pore size induced by variations in $\mathrm{pH}$. Polymers and Polymer Composites, 16, 359-364 (2008).

11 Debye, P. \& Hückel, E. The theory of electrolytes. I. Lowering of freezing point and related phenomena. Physikalische Zeitschrift 24, 185-206 (1923).

12 Hammann, C. H., Hamnett, A, \& Vielstich, W. Electrochemistry (Wiley-VCH Verlag $\mathrm{GmbH}$, Weinheim, 1998)

13 Harris, D. C. Quantitative Chemical Analysis 6th edn (W. H. Freeman \& Company, UK, 2003).

14 Verway, E. J. W. \& Overbeek, J. T. H. Theory of the Stability of Lyophobic Colloids (Elsevier, Amsterdam, 1948).

\section{APPENDIX}

\section{MATHEMATICAL MODEL}

For both lots of membranes the morphology is basically the same. The solution of polyacrylic acid are polyelectrolytes, in which the electric charge is due to the deprotonation of the acrylic group in the acid. The degree of deprotonation depends on the concentration of $\mathrm{H}^{+}$and is the responsible of the ionic strength; when this concentration changes, the polymer molecule suffers deformations caused by the competition of three main forces: Coulombian interactions due to the presence of electrical charge, steric interactions force produced by the overlap of polymer chains and the elastic forces due to entropic effects. Considering that the membrane is a solid polymer, it can suffer expansion or contraction according to its physicochemical properties. When the cellulose acetate and polyacrylic acid swell by the action of the water, they overlap each other, increasing the monomer concentration. An osmotic pressure appears and tries to separate them (steric interaction), as a consequence of which an elastic force appears because of the change in the conformational entropy, which tends to shrink its size. As mentioned earlier, the polyacrylic acid in water is a polyelectrolyte. The electric repulsion produces an expansion of the molecule and also contributes to the overlapping between molecules. Under this behavior, the double-layer overlapping, the steric interaction and the elastic force control the molecule size and consequently the pore size.

According to the Debye-Huckel approximation for charged particles in a continuous medium, the electric potential can be written as: ${ }^{11}$

$$
\Psi=\Psi_{\mathrm{o}} \mathrm{e}^{-k x}
$$

Where

$$
k^{2}=\frac{e}{\varepsilon k T} \sum_{i} z_{i}^{2} n_{i \mathrm{o}}
$$

$z_{i}$ is the valence number of the ions of type $i ; e$, the electron charge; $n_{i 0}$, the number concentration of ions of type $i$ per unit volume evaluated far from the charged molecule (bulk concentration); $\varepsilon$, the dielectric constant of the medium; $k$, the Boltzman constant; and $T$, the absolute temperature. $k^{-1}\left(\equiv \mathrm{l}_{\mathrm{D}}\right)$ is known as the 'Debye length of the electrical double layer'.

When two charged particles approach each other, the repulsion force $F_{\mathrm{DL}}$ per unit area produced by the overlapping of their electric double layers is as follows. ${ }^{13,14}$

$$
F_{\mathrm{DL}}=64 n_{\mathrm{o}} k T A_{\mathrm{o}}^{2} \mathrm{e}^{(-x / l d)}
$$

$A_{\mathrm{o}}$ is a constant that depends on geometry and $x$ is the separation between molecules.

A differential element of this force taken on a differential element of area $\mathrm{d} A=2 \pi x \mathrm{~d} x$ produces the next result:

$$
f_{\mathrm{DL}}=128 \pi n_{\mathrm{o}} k T A_{\mathrm{o}}^{2} \int \mathrm{e}^{\left(-x / l_{D}\right)} x \mathrm{~d} x
$$

Because at molecular level the distance between molecules is small after the integration of Equation (4) takes the shape:

$$
f_{\mathrm{DL}}=\operatorname{cte} x^{2}-O\left(x^{3}\right)
$$

Physically this result shows that when the particles approach each other the repulsion force depends quadratically with the separation distance (terms of higher order in Equation (5) are neglected).

Applying the Flory-Krigbaum theory, which predicts that the free energy has a linear dependence with the polymer volume fraction that is overlapped, it is possible to show that the polymer volume fraction 
can be written as: ${ }^{2}$

$$
\Phi_{x}(t)=\Phi_{\mathrm{o}}\left(\frac{y-y_{\min }}{y}\right)
$$

So, the force due to the steric interaction can be written as follows:

$$
f_{\text {steric }}=4 \pi \mathrm{RT}(\chi-1) N_{\mathrm{s}} \phi_{\mathrm{o}}\left(x^{2}-y_{\min } \frac{x^{2}}{y}\right)
$$

In this equation, $x$ and $y$ correspond to the changes in the pores and polymer dimensions, this means physically that when the polymer swells by the action of water the pore size is reduced, and when it deswells the pore size increase.

As has been explained through the analysis of the forces that interact in the behavior of the membranes, the total force can be written as:

$$
f_{\text {total }}=k_{1} x+k_{2} x^{2}-k_{3} \frac{x^{2}}{y}
$$

where the constants $k_{1}, k_{2}$ and $k_{3}$ are interpreted as the contribution of (a) the elastic force due to changes in the conformational entropy of the polymer chains, (b) the repulsion force produced by the overlap of the Debye's double layer and (c) the force due to the steric interactions produced by the concentrations gradients (overlap of molecules).

Taking into account that the molecules of the membrane are immersed in water and it can be considered as a viscous media, the total force felt by the molecules should be proportional to the velocity through the medium, then Equation (8) can be written as

$$
\frac{\mathrm{d} x}{\mathrm{~d} t}=k_{1} x+k_{2} x^{2}-k_{3} \frac{x^{2}}{y}
$$

The general solution of this differential equation is

$$
x(t)=\frac{x_{0}}{1+a \operatorname{Sin}^{2}(\omega t+\delta)}
$$

The physical interpretation of this equation is as follows: $x$ is the pore diameter at any time, $x_{0}$ is the initial value of the pore size, $a$ is an adimensional fitting constant, $\omega$ is the oscillation frequency, $\delta$ is the phase angle where the oscillation begins, $t$ is the time of thermal treatment. 